\title{
Statistical-Thermal Model Calculations using THERMUS
}

\author{
S. Wheaton, J. Cleymans \\ UCT-CERN Research Centre, Department of Physics, \\ University of Cape Town, \\ Rondebosch 7r01, Cape Town, South Africa
}

\begin{abstract}
Selected results obtained using THERMUS, a newly-developed statistical-thermal model analysis package, are presented.
\end{abstract}

\section{INTRODUCTION}

The statistical-thermal model has proved extremely successful in applications to relativistic collisions of both heavy ions and elementary particles (cf. 1] for recent surveys and references therein). In light of this success, THERMUS [2], a thermal model analysis package, has been developed for incorporation into the object-oriented ROOT framework 3]. This follows closely on the release of SHARE, a statistical hadronisation package, written in FORTRAN and Mathematica [4].

At present THERMUS treats the system quantum numbers $B, S$ and $Q$ within three distinct formalisms: a grand-canonical ensemble, in which the quantum numbers are conserved on average; a strangeness-canonical ensemble, in which strangeness is exactly conserved, while baryon content and charge are treated grand-canonically; and, finally, a fully canonical ensemble in which $B, S$ and $Q$ are each exactly conserved. A detailed theoretical overview of the statistical-thermal model as applicable to THERMUS, as well as a description of the structure of THERMUS, can be found in [2].

\section{STATISTICAL-THERMAL MODEL PREDICTIONS USING THERMUS}

In a chemical analysis within the grand-canonical ensemble, the statistical-thermal model requires 6 parameters as input: the chemical freeze-out temperature $T$, baryon-, strangeness- and charge chemical potentials $\mu_{B}, \mu_{S}$ and $\mu_{Q}$ respectively, strangeness saturation factor $\gamma_{S}$ and fireball volume $V$. When restricting one's attention to particle or density ratios, the volume dependence largely falls away, unlike in canonical treatments where the particle densities within these ensembles differ from those in the grand-canonical ensemble by correction factors which are strongly volume-dependent [5], as seen in Fig. 11.

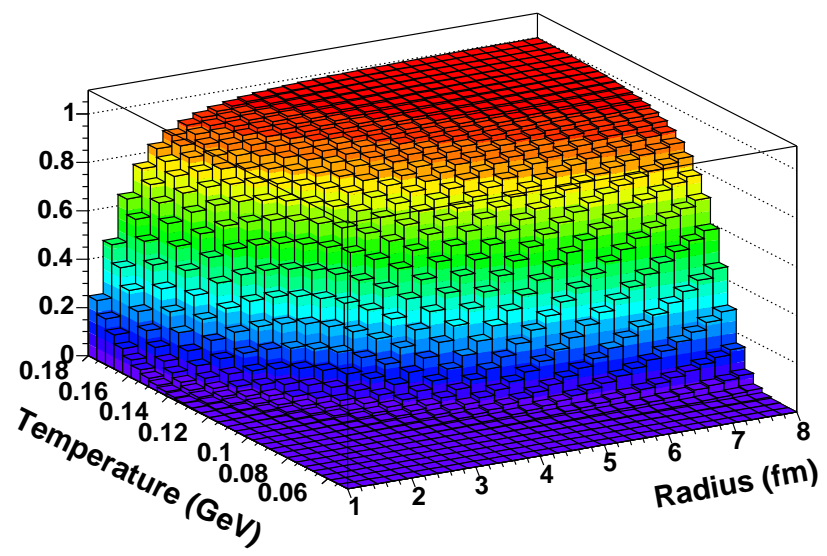

FIG. 1: The canonical correction factor of a $S=+2$ hadron in the strangeness-canonical ensemble as a function of the fireball temperature and radius, assuming full strangeness saturation and $S_{\text {fireball }}=\mu_{B}=\mu_{Q}=0$. 


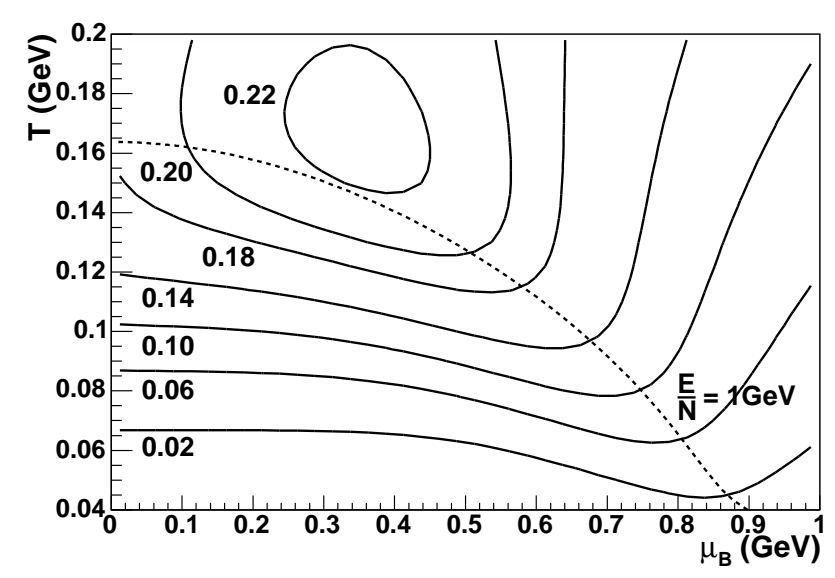

FIG. 2: Contour plot of the final $K^{+} / \pi^{+}$ratio in the $T-\mu_{B}$ plane with $\mu_{S}$ constrained to ensure strangeness neutrality, $\mu_{Q}=0$ and $\gamma_{S}=1$. Also shown as the dashed line is the freeze-out condition $E / N=1 \mathrm{GeV}$.

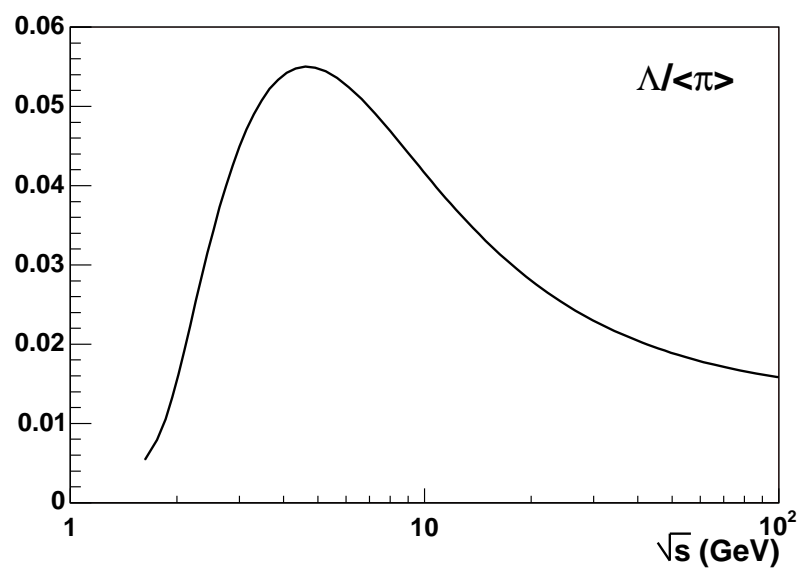

FIG. 3: The statistical-thermal model prediction for the $\Lambda /\langle\pi\rangle$ ratio generated by THERMUS.

Assuming full strangeness saturation (i.e. $\gamma_{S}=1$ ) and constraining the strangeness density and the ratio $B / 2 Q$ in the model leaves just $T$ and $\mu_{B}$ as free parameters. This allows all density and particle ratios to be plotted in the $T-\mu_{B}$ plane in which the freeze-out condition [6], $E / N=1 \mathrm{GeV}$, defines a curve $T\left(\mu_{B}\right)$ (Fig. [2).

From fits to experimental data it is possible to determine the energy dependence of the thermal parameters at chemical freeze-out, as done in [7]. In this way, the statistical-thermal model is able to make predictions as a function of collision energy. As an example, in Fig. [3 the THERMUS prediction for the $\Lambda /\langle\pi\rangle$ ratio, with $\langle\pi\rangle \equiv 3 / 2\left(\pi^{+}+\pi^{-}\right)$, is shown. In another application, one sees in Fig. 4 a breakdown in the feed-down contribution to the $\pi^{+}$. At low energy the majority of feeding comes from the baryon sector, while mesons dominate at high energy. This highlights the need for a thorough treatment of resonances within statistical-thermal models. The THERMUS distribution includes a text file listing all hadrons up to mass $2.6 \mathrm{GeV}$ with $u, d$ and $s$ quarks listed by the Particle Data Group [8], as well as text files listing the particle decay channels. 


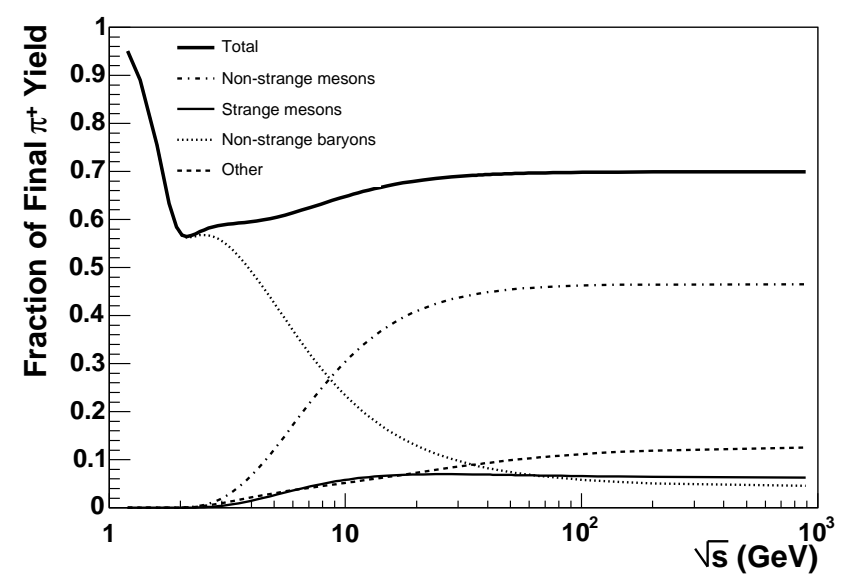

FIG. 4: The decay contribution to the $\pi^{+}$from various sources (weak decays excluded).

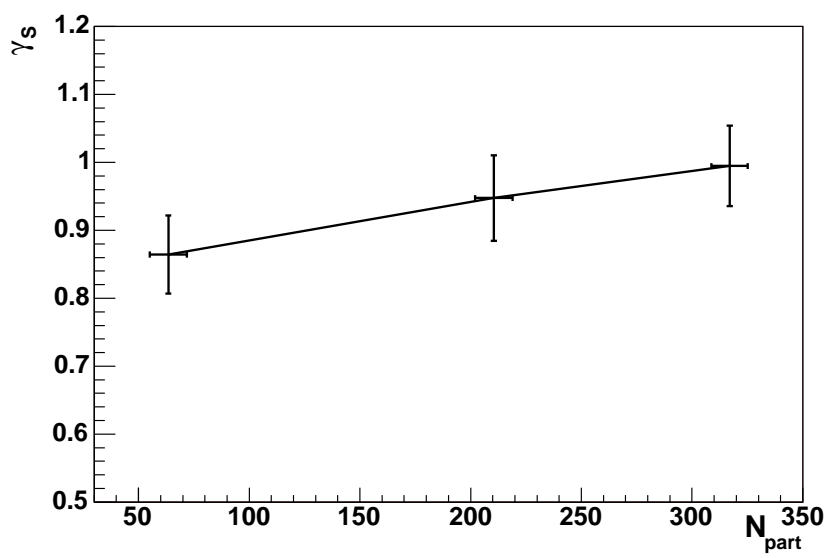

FIG. 5: The centrality dependence of the strangeness saturation factor, $\gamma_{S}$, as extracted from $\mathrm{Au}+\mathrm{Au}$ data at $\sqrt{s_{N N}}=130$ $\mathrm{GeV}$ [9].

\section{THERMAL FITS WITHIN THERMUS}

Fits to experimental data are easily accomplished within THERMUS. Since feed-down corrections and reconstruction efficiencies are often particle- and experiment specific, THERMUS is capable of ascribing to each particle yield or ratio of interest its own particle set and associated decay chain (or separate sets for the numerator and denominator in the case of ratios). Use is made of the ROOT TMinuit fitting class.

In a recent application of THERMUS [9], particle ratios measured in $\mathrm{Au}+\mathrm{Au}$ collisions at $\sqrt{s_{N N}}=130 \mathrm{GeV}$ by all four RHIC experiments, and converted to a common centrality binning in [10], were analysed. A grand-canonical treatment was employed with quantum statistics and resonance width included for all particles, $\mu_{Q}=0$, and $T, \mu_{B}$, $\mu_{S}$ and $\gamma_{S}$ as fit parameters. While the temperature and chemical potentials were found to be largely centrality independent, the strangeness saturation factor, $\gamma_{S}$, was observed to increase with participant number towards unity in central collisions (Fig. [5. This confirmed previous findings at SPS and RHIC energies [10, 11] that deviations from equilibrium conditions diminish with increasing collision centrality. 


\section{CONCLUSION}

In conclusion, an analysis package, THERMUS, has been developed allowing thermal model analyses to be performed within the ROOT framework. Within THERMUS statistical-thermal model calculations are possible within three distinct formalisms with a complete treatment of hadronic resonances up to a mass of $2.6 \mathrm{GeV}$. Fits to experimental data are furthermore easily achieved.

THERMUS is available freely from http://hep.phy.uct.ac.za/THERMUS/, along with a detailed user guide and installation instructions.

\section{ACKNOWLEDGEMENTS}

We acknowledge the support of the National Research Foundation (NRF, Pretoria) and the URC and PGSO of the University of Cape Town.

[1] P. Braun-Munzinger, K. Redlich, J. Stachel, nucl-th/0304013 in Quark-Gluon Plasma 3, (eds.) R. Hwa, X.N. Wang; K. Redlich, J. Cleymans, H. Oeschler and A. Tounsi, Acta Phys. Polonica B 33 (2002) 1609;

J. Letessier, J. Rafelski, Int. J. Mod. Phys. E 9 (2000) 107.

[2] S. Wheaton and J. Cleymans, hep-ph/0407174

[3] R. Brun and F. Rademakers, Nucl. Inst. \& Meth. in Phys. Res. A 389 (1997) 81.

See also http://root.cern.ch/

[4] G. Torrieri, S. Steinke, W. Broniowski, W. Florkowski, J. Letessier, J. Rafelski, nucl-th/0404083

[5] A. Keränen and F. Becattini, Phys. Rev. C 65 (2002) 044901, Erratum-ibid. C 68 (2003) 059901 and references therein.

[6] J. Cleymans and K. Redlich, Phys. Rev. Lett. 81 (1998) 5284;

J. Cleymans and K. Redlich, Phys. Rev. C 60 (1999) 054908.

[7] P. Braun-Munzinger, J. Cleymans, H. Oeschler and K. Redlich, Nucl. Phys. A 697 (2002) 902.

[8] K. Hagiwara et al., Phys. Rev. D 66 (2002) 010001.

[9] J. Cleymans, B. Kämpfer, M. Kaneta, S. Wheaton and N. Xu, hep-ph/0409071

[10] M. Kaneta and N. Xu, nucl-th/0405068

[11] J. Cleymans, B. Kämpfer, S. Wheaton, Phys. Rev. C 65 (2002) 027901;

J. Cleymans, B. Kämpfer, S. Wheaton, Nucl. Phys. A 715 (2003) 553c;

J. Cleymans, B. Kämpfer, P. Steinberg, S. Wheaton, hep-ph/0212335 J. Phys. G 30 (2004) S595;

B. Kämpfer, J. Cleymans, K. Gallmeister, S. Wheaton, hep-ph/0202134 Acta. Phys. Hung. New Ser. Heavy Ion Phys. 18 (2003) 1. 